\title{
The role of PAQR3 gene promoter hypermethylation in breast cancer and prognosis
}

\author{
JINPENG CHEN*, FEIRAN WANG*, JUNFEI XU*, ZHIXIAN HE, YUHUA LU and ZHIWEI WANG \\ Department of General Surgery, The Affiliated Hospital of Nantong University, Nantong, Jiangsu 226001, P.R. China
}

Received February 2, 2015; Accepted April 1, 2016

DOI: 10.3892/or.2016.4951

\begin{abstract}
PAQR3 is a tumor suppressor in breast cancer and its expression regulation mechanism has not been well elucidated. In this study, we found that PAQR3 expression was downregulated in breast cancer tissues, and the downregulation of PAQR3 expression was found to be significantly associated with aberrant methylation of the gene promoter. Methylation-specific PCR showed that hypermethylation of the PAQR3 gene was observed in $71.8 \%$ of the breast cancers, whereas it was found in only $28.2 \%$ of the corresponding non-tumor tissues. Moreover, we found that the PAQR3 promoter methylation status was related to lymph node metastasis $(\mathrm{P}=0.01)$. In addition, overexpression of PAQR3 inhibited breast cancer cell invasion and growth. Furthermore, PAQR3 expression was restored in MCF-7 cells after treatment with the demethylating agent, 5-aza-2'-deoxycytidine, and the effect of demethylation induced invasion and proliferation suppression of MCF-7 cells. Collectively, our results suggested that the aberrant methylation of PAQR3 underlies its downregulation in breast cancer and our data indicated that epigenetic silencing of PAQR3 gene expression by promoter hypermethylation may play an important role in breast cancer.
\end{abstract}

\section{Introduction}

Breast cancer, a leading type of cancer occurring in women, tends to invade into adjacent regions and to metastasize to lymph nodes and adjacent organs (1). During the process of metastasis, tumor-suppressor genes are inactivated, which may be responsible for breast cancer metastasis (2). Promoter hypermethylation is a type of epigenetic alteration associated with gene silencing. Aberrant hypermethylation of tumor suppressor genes is an important epigenetic event in the development and progression of breast cancer (3).

Correspondence to: Professor Zhiwei Wang, Department of General Surgery, The Affiliated Hospital of Nantong University, 20 Xisi Road, Nantong, Jiangsu 226001, P.R. China

E-mail: youyi701@163.com

*Contributed equally

Key words: breast cancer, PAQR3, methylation, prognosis
PAQR3, also known as Raf kinase trapping to Golgi (RKTG), belongs to the family of progestin and adipoQ receptor (PAQR) and is a seven-transmembrane protein specifically localized in the Golgi apparatus in mammalian cells $(4,5)$. Subsequent characterization of PAQR3 indicates that it might negatively modulate Ras-mediated signaling by isolating Raf kinase to the Golgi apparatus (4). PAQR3 acts as a tumor suppressor mainly via its inhibitory activity on Raf/MEK/ERK signaling (6-8). For example, the PAQR3 expression level was markedly decreased in colorectal cancer samples compared with adjacent normal tissues and the expression level of PAQR3 was inversely associated with tumor grade in colorectal cancer samples (9). PAQR3 also inhibited cell proliferation, migration, sprouting and angiogenesis of endothelial cells, and the expression level of PAQR3 was found to be significantly downregulated in clinical clear-cell renal cell carcinoma samples, with an inverse correlation with VEGF expression level (7). Furthermore, it was reported that PAQR3 was downregulated in gastric cancer and was closely associated with metastasis progression and survival in patients with gastric cancer (10). Moreover, some data strongly suggest that downregulation of PAQR3 promotes tumor metastasis and proliferation through induction of ERK phosphorylation in osteosarcoma (11). Recently, it has been reported that PAQR3 expression is downregulated in human breast cancers and plays an important role in carcinogenesis. However, the molecular mechanisms of expression regulation that lead to tumorigenesis and progression of breast cancer are still not clearly understood.

In the present study, we reported that PAQR3 is important for breast tumorigenesis. Decreased PAQR3 expression was observed in breast cancer patient samples. PAQR3 overexpression in breast cancer cells inhibited tumor growth and invasion in vitro. We examined the methylation status of PAQR3 in breast cancer and matched non-tumor samples and determined whether promoter methylation was associated with decreased gene expression in breast cancer cell lines. We also examined associations between PAQR3 methylation and several clinicopathological parameters, and concluded that PAQR3 reactivation is associated with demethylation of the PAQR3 gene by 5-Aza-dC treatment in MCF-7 cells.

\section{Materials and methods}

Tissue samples and cell lines. A total of 46 breast cancer tissues and paired adjacent non-tumor tissues obtained from surgically resected specimens at the Affiliated Hospital of 
Nantong University during the period from 2010 to 2011 were analyzed. All specimens were immediately frozen in liquid nitrogen and kept at $-80^{\circ} \mathrm{C}$ until RNA and protein extractions were performed. None of the patients had received any preoperative adjuvant therapy. The resected tissue specimens from these patients were fixed in $10 \%$ formalin and embedded in paraffin. Written informed consent was obtained from all patients. The study was approved by the Ethics Committee of the Affiliated Hospital of Nantong University.

Human breast cancer cell lines MCF-7, MDA-MB-231, MDA-MB-453, MDA-MB-468 and T47D were cultured in medium supplemented with $10 \%$ fetal bovine serum (FBS) (Gibco) at $37^{\circ} \mathrm{C}$ in a $5 \% \mathrm{CO}_{2}$ incubator. For overexpression of endogenous PAQR3, the coding sequence of PAQR3 was amplified and subcloned into the pcDNA3.1(+) vector (Invitrogen, Carlsbad,CA,USA) according to the manufacturer's instructions.

Real-time quantitative PCR. Total RNA was extracted using the TRIzol protocol (Invitrogen). cDNA was subsequently synthesized from total RNA using an Omniscript RT kit (Qiagen, Valencia, CA, USA). A $25-\mu 1$ reaction mixture contained $1 \mu \mathrm{l}$ of cDNA from samples, $12.5 \mu \mathrm{l}$ of $2 \mathrm{X}$ Fast EvaGreen $^{\mathrm{TM}}$ qPCR Master Mix, $1 \mu$ l primers $(10 \mathrm{mM})$, and $10.5 \mu \mathrm{l}$ of RNase/DNase-free water. The amplification conditions for 40 cycles consisted of denaturation at $96^{\circ} \mathrm{C}$ for $2 \mathrm{~min}$, annealing at $96^{\circ} \mathrm{C}$ for $15 \mathrm{sec}$, and extension at $60^{\circ} \mathrm{C}$ for $1 \mathrm{~min}$. Quantitative PCR analysis was then performed for PAQR3 mRNA expression, and data were normalized to GAPDH levels and determined by the $2^{-\Delta \Delta \mathrm{Ct}}$ method. All analyses were performed using Eppendorf Mastercycler ${ }^{\circledR}$ ep realplex $(2 S$; Eppendorf, Hamburg, Germany). The sequences of the primers for PAQR3 were as follows: PAQR3 forward, 5'-TTCAAGACC CACATCAACC-3' and reverse, 5'-TTTCCCTTGTATTTC CATTC-3'.

Western blotting. Total protein was extracted by lysis buffer containing protease inhibitors (Promega, Madison, WI, USA). Equal amounts of protein were separated by $12 \%$ polyacrylamide gel electrophoresis and then transferred to a polyvinylidene fluoride (PVDF) membrane. After being blocked with $5 \%$ non-fat milk, the membrane was incubated with the primary antibodies overnight at $4^{\circ} \mathrm{C}$. A goat polyclonal antibody against PAQR3 (1:500; Santa Cruz Biotechnology, USA) was used and membranes were washed three times in TBST for $5 \mathrm{~min}$ and subsequently incubated with a secondary antibody, anti-goat IgG-conjugated IRDye 800 (1:5,000; Rockland, Gilbertsville, PA, USA) at room temperature for $2 \mathrm{~h}$, followed by scanning with an Odyssey Infrared Imaging system (LI-COR Biosciences, Lincoln, NE, USA), and analyzed with PDQuest 7.2.0 software (Bio-Rad). $\beta$-actin was used as the loading control.

Methylation-specific PCR and bisulfite sequencing PCR. The methylation status of the PAQR3 promoter was determined by methylation-specific PCR (MSP) and bisulfite sequencing PCR (BSP). Genomic DNA extracted from tissues and cells was modified with bisulfite reagents following the manufacturer's instructions. This modification converts unmethylated cytosine to thymine, whereas methylated cytosine remained unchanged. PCR amplification was performed using $2.0 \mu \mathrm{l}$ of bisulfite-modified DNA in a total volume of $50 \mu 1$ reaction containing $2 \mu \mathrm{l}$ of each primer, $5 \mu \mathrm{l}$ of 10X DreamTaq Buffer, and $2.0 \mathrm{mM}$ dNTP Mix and 1.25 U DreamTaq (Fermentas). The MSP conditions were as follows: $94^{\circ} \mathrm{C}$ for $5 \mathrm{~min}, 40$ cycles of $94^{\circ} \mathrm{C}$ for $30 \mathrm{sec}, 60^{\circ} \mathrm{C}$ for $30 \mathrm{sec}, 72^{\circ} \mathrm{C}$ for $45 \mathrm{sec}$ and $72^{\circ} \mathrm{C}$ for $10 \mathrm{~min}$. Methylation-specific PCR products were analyzed by a $2 \%$ agarose gel. Forward and reverse primers for the methylated sequence (M) were 5'-TTGTTGAAGAGCGCG TATTATATC-3' and 5'-TAAAAAACCCGAAAATCTACT CGTA-3', respectively, and for the unmethylated sequence (U) 5'-TTGTTGAAGAGTGTGTATTATATTGA-3' and 5'-TAA AAAACCCAAAAATCTACTCATA-3', respectively. Moreover, amplified MSP products were analyzed using BioEdit and ClustalW alignment tools. The BSP conditions were as follows: $94^{\circ} \mathrm{C}$ for $5 \mathrm{~min}, 40$ cycles of $94^{\circ} \mathrm{C}$ for $30 \mathrm{sec}$, $56^{\circ} \mathrm{C}$ for $30 \mathrm{sec}, 72^{\circ} \mathrm{C}$ for $45 \mathrm{sec}$ and $72^{\circ} \mathrm{C}$ for $10 \mathrm{~min}$, and directly sequenced using the ABI 3700 automated sequencing system (Applied Biosystems, Foster City, CA, USA). Forward and reverse primers for BSP were 5'-GATGTATTAGAA GTTGTTGAAGAG-3' and 5'-AACAAAAAAAATATAAAT AAAAAAAA-3', respectively.

Treatment of cells with 5-Aza-dC. MCF-7 cells were seeded at a density of $5 \times 10^{5}$ cells/well in 6-well culture plates in Dulbecco's modified Eagle's medium (DMEM) (Invitrogen) containing $10 \% \mathrm{FBS}$, and incubated in a humidified atmosphere of $5 \% \mathrm{CO}_{2}$ at $37^{\circ} \mathrm{C}$. After overnight culture, the cells were incubated with medium containing 0,5 and $10 \mu \mathrm{mol} / 1$ 5-aza-2'-deoxycytidine (5-Aza-dC) (Sigma, St. Louis, MO, USA) for 3 days. Total RNA and protein were isolated after treatment, and PAQR3 mRNA and protein were analyzed by qPCR and western blotting as aforementioned.

Cell invasion assay. For the invasion assays, the cells were suspended in serum-free medium and plated in duplicate in the top well of Matrigel invasion chambers (8-mm pore size; Corning, Inc., Corning, NY, USA). Complete medium was placed in the lower chamber and cells were allowed to invade for $24 \mathrm{~h}$ at $37^{\circ} \mathrm{C}$ in $5 \% \mathrm{CO}_{2}$. Non-invading cells in the upper chamber were removed using a cotton swab, and cells on the lower chamber were fixed in methanol and stained with $0.1 \%$ crystal violet. The number of invasive cells was counted in three random fields per experiment from three independent experiments.

Colonyformation assay. Cells were seeded to $1.0 \times 10^{3}$ cells/well in 6-well plates and cultured in DMEM medium for 3 weeks at $37^{\circ} \mathrm{C}$ in $5 \% \mathrm{CO}_{2}$. Surviving colonies were stained with $0.5 \%$ crystal violet and visible colonies were counted. All the experiments were performed in triplicate wells three times.

Statistical analysis. All quantified data represent an average of at least triplicate samples. SPSS 17.0 (SPSS, Inc., Chicago, IL, USA) was used for statistical analysis. Data are presented as means \pm SD. One-way ANOVA or two-tailed Student's t-test was used for comparisons between groups. A chi-square test or Fischer's exact test was used to identify differences between categorical variables. Survival analysis was performed using the Kaplan-Meier method. $\mathrm{P}<0.05$ was considered to indicate statistically significant results. 

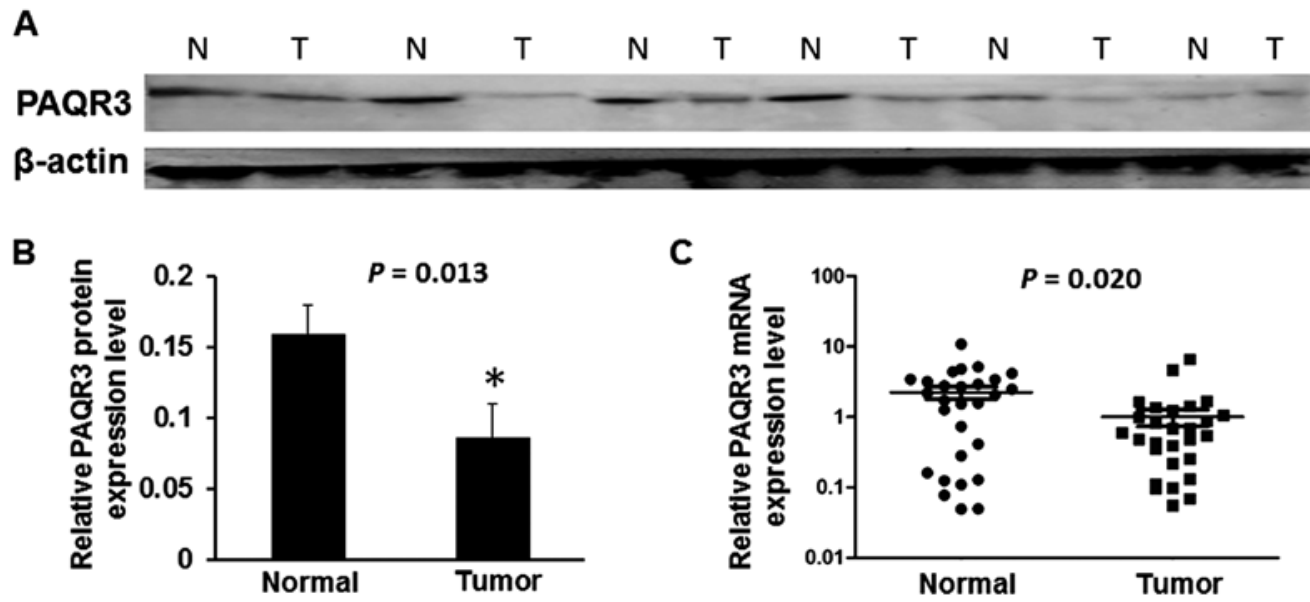

Figure 1. Expression of PAQR3 in breast cancer tissues was evaluated by western blotting and real-time quantitative PCR. (A) Representative results of PAQR3 protein expression in six paired breast cancer $(\mathrm{T})$ and matched adjacent non-tumor $(\mathrm{N})$ tissues. (B) Relative PAQR3 protein expression level was markedly decreased in 22 of the 28 cases compared with the corresponding adjacent non-tumor tissues ( $\mathrm{P}=0.013)$. (C) The relative mRNA expression of PAQR3 was significantly decreased in breast cancer tissues compared to matched adjacent non-tumor tissues $(\mathrm{n}=28)$ as assessed by qPCR $(\mathrm{P}=0.020)$.

A

C

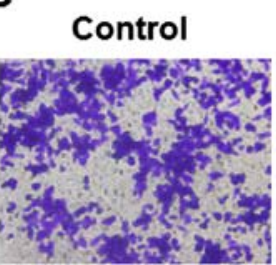

E

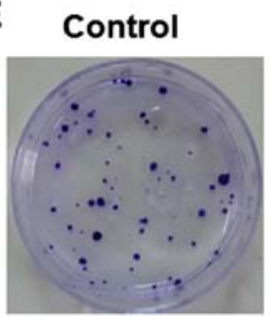

Vector

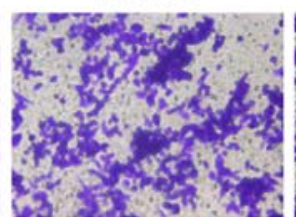

Vector

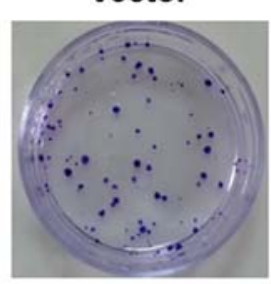

PAQR3

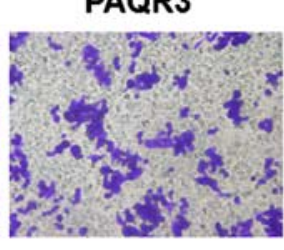

PAQR3

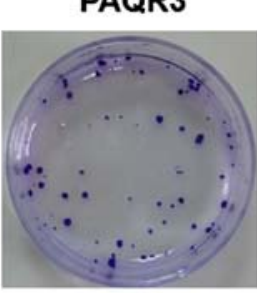

B
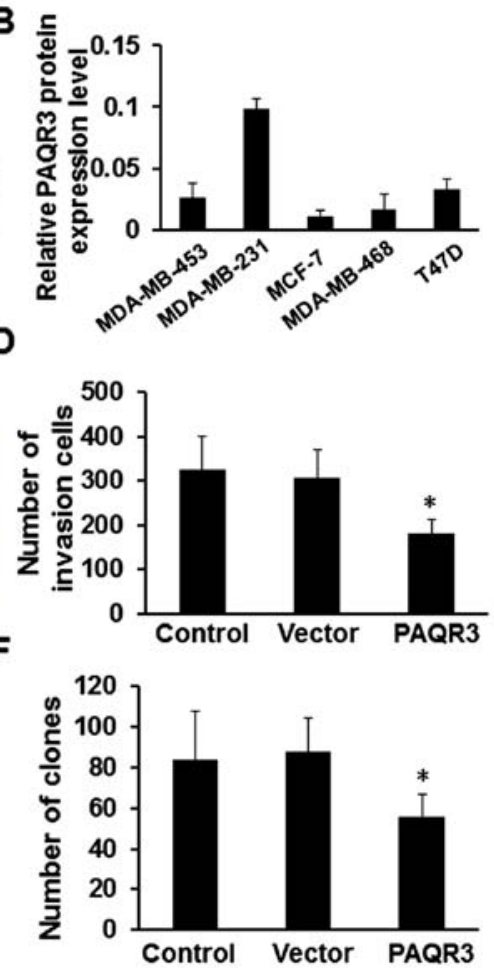

Figure 2. Overexpression of PAQR3 inhibits invasion and growth in vitro. (A and B) The expression of PAQR3 was detected in five breast cancer cell lines. The highest expression was detected in the MDA-MB-231 cell line and the lowest PAQR3 expression was detected in the MCF-7 cell line when compared with the other breast cancer cell lines. (C and D) Overexpression of PAQR3 inhibited cell invasion in the MCF-7 cells as demonstrated by Transwell assays ("P $<0.05$ ). Representative images of stained cells are shown with the original magnification of x100. (E and F) Colony numbers of the pcDNA3.1 (+)-PAQR3 or empty vector and control cells in plate assays ( $\mathrm{P}<0.05)$. The data are presented as the means \pm SEM of three independent experiments.

\section{Results}

Expression of PAQR3 is downregulated in breast cancer tissues. In order to assess the expression of PAQR3 in breast cancer tissues, we performed real-time PCR and western blotting to measure the expression of PAQR3 mRNA and protein in 28 freshly collected breast cancer tissues and corresponding paracancerous normal tissues. We found that the average relative expression of PAQR3 mRNA was markedly downregulated compared to that in the corresponding adjacent non-cancerous normal tissues $(\mathrm{P}=0.020$, Fig. $1 \mathrm{C})$. We also found, compared with adjacent non-tumor tissues, that the level of PAQR3 protein in breast cancer tissues was significantly decreased in 22 of the 28 cases ( $\mathrm{P}=0.013$, Fig. 1B). Six cases represented the control non-tumor tissues (Fig. 1A).

Overexpression of PAQR 3 inhibits cell invasion and cell colony formation in vitro. We first detected the expression 


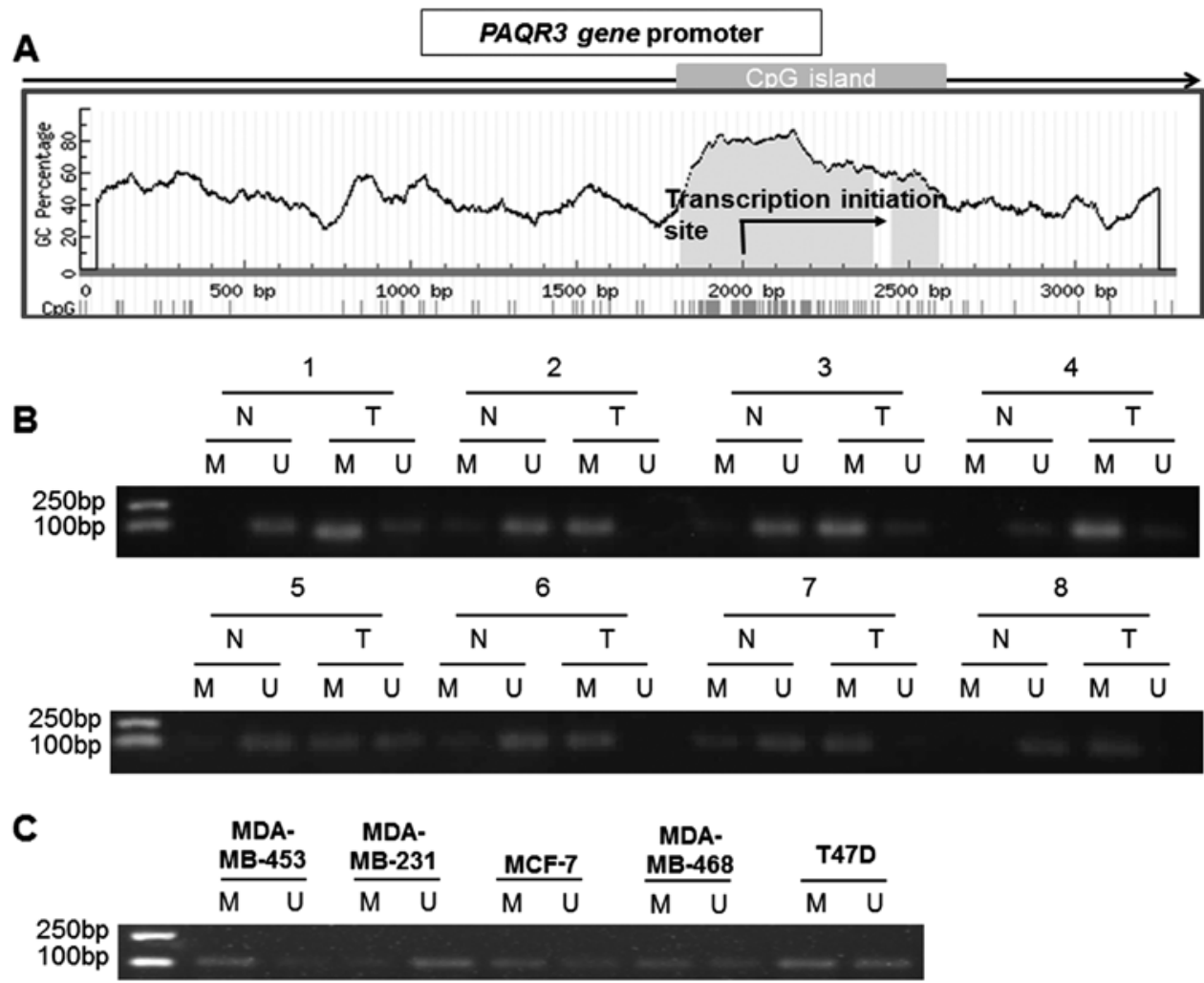

Figure 3. Methylation-specific PCR analysis showed PAQR3 promoter methylation. (A) Schematic depiction of the PAQR3 promoter-associated CpG islands, which span the region from -188 to +587 . (B) Representative examples of the methylation analysis of PAQR 3 in breast cancer (T) and their corresponding non-tumor (N) tissues. (C) Methylation levels of PAQR3 in the cell lines were also detected by MSP. The promoter of the GRK6 gene was hypermethylated in the MCF-7 and MDA-MB-453 cells, and hypomethylated in the MDA-MB-231 cells. M, methylated; U, unmethylated.

level of the PAQR3 protein in cell lines, and five breast cancer cell lines were analyzed using western blotting. The expression of PAQR3 was detected in all cell lines and the lowest PAQR3 expression level was detected in the MCF-7 cell line when compared with the other breast cancer cell lines (Fig. 2A and B). In the present study, we chose the MCF-7 cells for further investigation, and then, we evaluated the potential role of PAQR3 on cellular invasion by Transwell assays in the MCF-7 cell line. MCF-7 cells were transfected with the PAQR3 overexpressing or empty vector plasmid and seeded in the chamber and their invasion abilities were determined $48 \mathrm{~h}$ later. The results showed that overexpression of $\mathrm{PAQR} 3$ was associated with a significant reduction of invasion ability compared to the empty vector (Fig. $2 \mathrm{C}$ and $\mathrm{D}, \mathrm{P}<0.05$ ). We further evaluated the potential effect of PAQR3 on cell colony formation and found that the overexpression of PAQR3 significantly inhibited the colony formation of the MCF-7 cells (Fig. 2E and F, P<0.05).

Promoter hypermethylation is involved in decreased PAQR3 expression. It has been reported that hypermethylation is one of the main causes of decreased gene expression in most types of cancer. To identify whether reduced PAQR3 expression is due to promoter hypermethylation, we studied the methylation status of the PAQR3 gene promoter in breast cancer. We searched for $\mathrm{CpG}$ islands in the PAQR3 gene promoter by using the online accessible software MethPrimer (Fig. 3A). Then we analyzed the methylation status of the PAQR3 gene promoter in cancerous tissue samples and their paired adjacent non-tumor tissues from 46 breast cancer patients by using MSP analysis. As expected, we observed that hypermethylation of PAQR3 was detected in $71.8 \%$ (33/46) of the breast cancer tissues, and $28.2 \%$ (13/46) of the adjacent non-tumor tissues. The difference in PAQR3 methylation between breast cancer and adjacent non-tumor tissue specimens was significant $(\mathrm{P}<0.001)$. Representative MSP results are shown in Fig. 3B, and the first to the sixth sample in Fig. 3B correspond to the six cases in Fig. 1A. We also examined the DNA methylation status of the PAQR3 gene promoter in breast cancer cell lines. Data from MSP analysis showed hypermethylation of the PAQR 3 gene promoter in the MCF-7 and MDA-MB- 453 cells, and hypomethylation in the MDA-MB-231 cells (Fig. 3C).

To further illustrate the methylation status of the PAQR3 promoter, we performed bisulfite sequencing around the promoter region of the $\mathrm{PAQR} 3$ gene in some of the breast cancer tissues. Specific primers without $\mathrm{CpG}$ sites were used to amplify the region spanning position from +204 to +447 . The sequence including the $15 \mathrm{CpG}$ sites is shown in Fig. 4A. Bisulfite sequencing of 10 individual clones of PCR products from breast cancer tissues revealed densely methylated CpGs within the promoter region compared with the non-tumor tissues ( $\mathrm{P}<0.05$, Fig. 4B). These results indicated that hypermethylation may be involved in the transcriptional repression of PAQR3.

$P A Q R 3$ promoter methylation is associated with clinicopathological features and poor prognosis. The correlations between methylation of PAQR3 and the clinicopathologic features of 
A

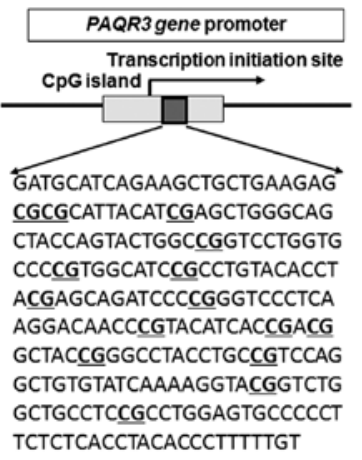

B

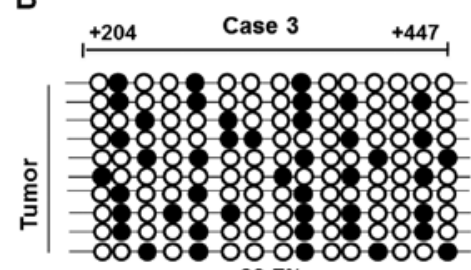

$28.7 \%$

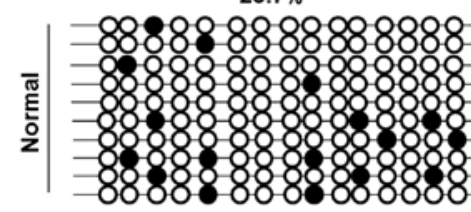

$11.3 \%$

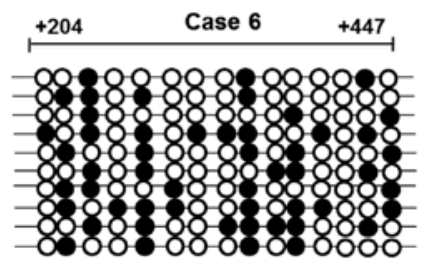

$34.7 \%$

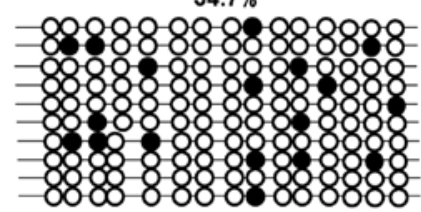

$12 \%$

Figure 4. Bisulfite sequencing analysis was performed in the $\mathrm{CpG}$ islands in the promoter of the PAQR3 gene. (A and B) Methylation status of $\mathrm{CpG}$ sites around the PAQR3 promoter region were analyzed in breast cancer and adjacent non-tumor tissues. Region spans from +204 to +447 including the $15 \mathrm{CpG}$ sites. Each row represents an individual subclone. Methylated and unmethylated $\mathrm{CpG}$ sites are shown as black circles and white circles, respectively.

Table I. Clinicopathological parameters of the breast cancer samples and PAQR3 methylation.

\begin{tabular}{|c|c|c|c|c|}
\hline \multirow[b]{2}{*}{$\begin{array}{l}\text { Clinical } \\
\text { parameters }\end{array}$} & \multirow[b]{2}{*}{$\begin{array}{l}\text { Total } \\
(\mathrm{n}=46)\end{array}$} & \multicolumn{2}{|c|}{ PAQR3 expression } & \multirow[b]{2}{*}{ P-value } \\
\hline & & $\begin{array}{l}\text { Methylated } \\
\quad(\mathrm{n}=33)\end{array}$ & $\begin{array}{l}\text { Unmethylated } \\
\quad(\mathrm{n}=13)\end{array}$ & \\
\hline Age (years) & & & & 0.068 \\
\hline$<50$ & 22 & 13 & 9 & \\
\hline$\geq 50$ & 24 & 20 & 4 & \\
\hline $\begin{array}{l}\text { Tumor size } \\
(\mathrm{cm})\end{array}$ & & & & 0.278 \\
\hline$<2$ & 19 & 12 & 7 & \\
\hline$\geq 2$ & 27 & 21 & 6 & \\
\hline $\begin{array}{l}\text { Lymph node } \\
\text { metastasis }\end{array}$ & & & & $0.010^{b}$ \\
\hline Positive & 21 & 19 & 2 & \\
\hline Negative & 25 & 14 & 11 & \\
\hline TNM stage & & & & 0.057 \\
\hline $\mathrm{I}+\mathrm{II}$ & 29 & 18 & 11 & \\
\hline III & 17 & 15 & 2 & \\
\hline ER status & & & & 0.667 \\
\hline Positive & 26 & 18 & 8 & \\
\hline Negative & 20 & 15 & 5 & \\
\hline PR status & & & & 0.887 \\
\hline Positive & 24 & 17 & 7 & \\
\hline Negative & 22 & 16 & 6 & \\
\hline $\begin{array}{l}\text { HER2/neu } \\
\text { status }\end{array}$ & & & & 0.161 \\
\hline Positive & 18 & 15 & 3 & \\
\hline Negative & 28 & 18 & 10 & \\
\hline
\end{tabular}

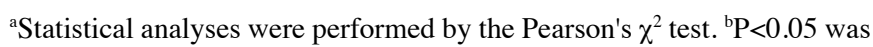
considered statistically significant.

these patients are summarized in Table I. We found that the PAQR3 gene promoter methylation status was related to tumor lymph node metastasis $(\mathrm{P}=0.010)$, but there was no significant difference in clinicopathologic features, including age, tumor size, TNM stage, ER, PR and HER2/neu status between methylated and unmethylated tumors from these patients. Moreover, hypermethylation of the PAQR3 promoter in breast cancer was significantly correlated with poor prognosis by Kaplan-Meier curve analysis with the log-rank test $\left(\chi^{2}=4.598\right.$, $\mathrm{P}=0.032$, Fig. 6).

Reactivation of PAQR3 expression after treatment with 5-Aza- $d C$. To further confirm that aberrant methylation was responsible for suppressing PAQR3 expression, we treated the MCF-7 breast cancer cells with the demethylating agent 5-Aza-dC. We found that the expression of PAQR3 mRNA and protein was significantly elevated in the MCF-7 cells with the highest expression occurring at a concentration of $10 \mu \mathrm{mol} / 1$ (Fig. 5A-C). Moreover, to further detect whether the reactivation of PAQR3 expression can regulate breast cancer proliferation and invasion, we analyzed the capability of growth and invasion in the MCF-7 cells using colony formation and Transwell assays. The Transwell assay showed that the number of invading cells that were treated with 5-Aza-dC was markedly reduced compared with the untreated group (Fig. 5D and $\mathrm{E}, \mathrm{P}<0.05$ ). A significant reduction in colony numbers was observed in the MCF-7 cells treated with 5-Aza-dC, compared with the control untreated group in the plate assays (Fig. 5F and G, $\mathrm{P}<0.05$ ). Thus, it is clear that the colony formation rate and invasive capacity of MCF-7 cells treated with 5-Aza-dC was markedly decreased compared with the untreated group as a result of the effect of demethylation.

\section{Discussion}

The genomic map of the PAQR3 gene, which resides on chromosome 4q21.21 and encodes one protein in most species, has been described (4). In contrast to the other PAQR family members, PAQR3 diverged quite early from the family and exhibited relatively independent differentiation (12). On the one hand, PAQR3 was proven to regulate cell proliferation and invasion by inhibiting ERK phosphorylation. On the other hand, PAQR3 can block cell proliferation and survival due to 


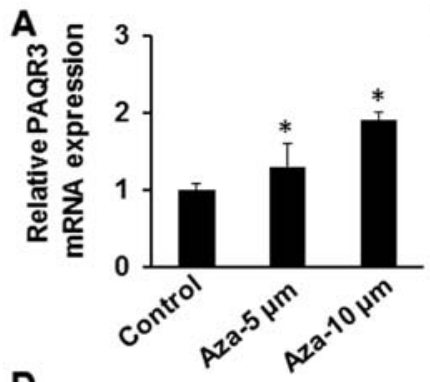

D

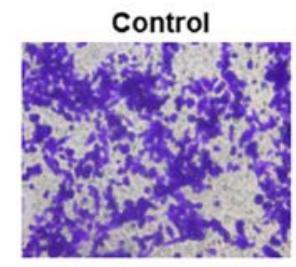

$\mathbf{F}$

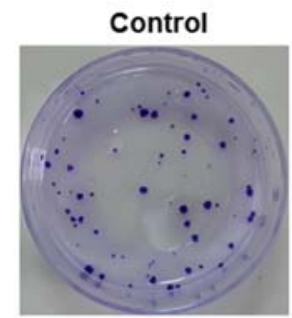

B

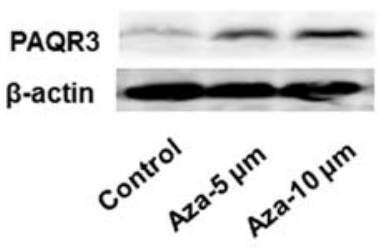

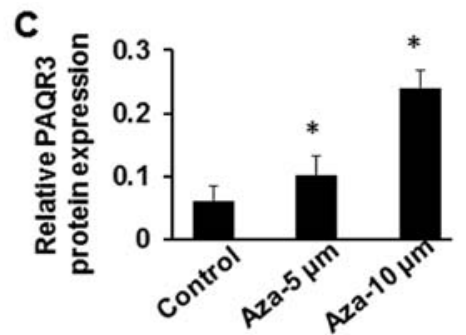

E

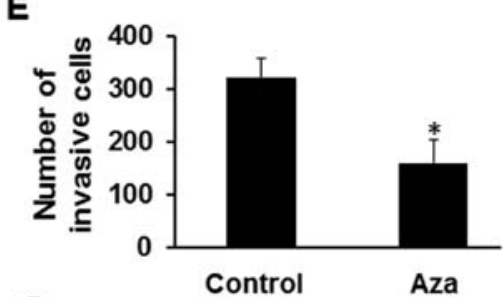

G
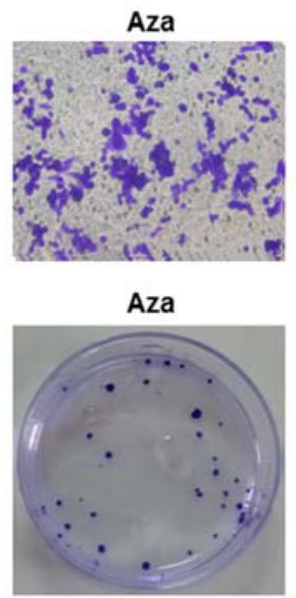

G

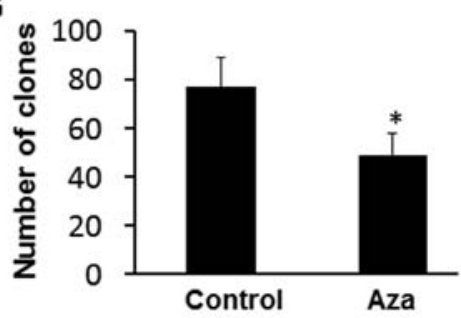

Figure 5. Downregulation of PAQR3 in the MCF-7 cells is associated with methylation of PAQR3. (A-C) qPCR and western blotting of PAQR3 expression after treatment with 5-Aza-dC (Aza) (5 or $10 \mu \mathrm{M})$ in the MCF-7 cells. (D and E) The Matrigel invasion assay was performed to assess the demethylation effect of PAQR3 on the invasion ability of the MCF-7 cell line after treatment with 5-Aza-dC and showed that the number of invading cells was significantly reduced after treatment with 5-Aza-dC compared with the control group ( $\mathrm{P}<0.05)$. ( F and G) Clone formation rate of the MCF-7 cells after treatment with 5-Aza-dC $(10 \mu \mathrm{M})$ was assessed by the colony formation assay ("P<0.05). The columns indicate the number of clones at the $24-\mathrm{h}$ time point. Results showed that the clone number of MCF-7 cells was significantly decreased after treatment with 5-Aza-dC compared with the control group.

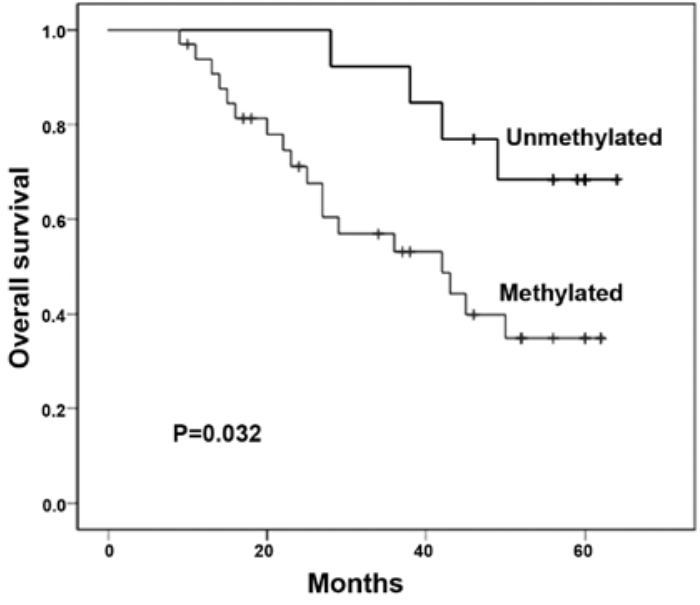

Figure 6. Kaplan-Meier analysis of overall survival of breast cancer patients with or without PAQR3 promoter methylation in breast cancer tissues.

the negative regulation of the Ras/MAPK and the PI3K/Akt pathways, or promote tumor metastasis and proliferation through induction of ERK phosphorylation $(4,11,13)$. Hence, we determined that the expression level of PAQR3 is closely associated with the progression and metastasis of cancers (9). Collectively, these findings indicated that PAQR3 is actively implicated in the regulation of cell proliferation and migration and plays an important role in tumor development $(6,14)$. In the present study, the results from qPCR and western blotting showed that PAQR 3 mRNA and protein expression was significantly downregulated in breast cancer tissues demonstrating that PAQR3 acted as a tumor suppressor gene, as previously reported in other studies as well (15). We also detected the expression level of the PAQR 3 protein in five breast cancer cell lines by western blotting, and found that the lowest PAQR3 expression level was detected in the MCF-7 cell line, and the highest expression level in the MDA-MB-231 cells. However, the proliferation and migration potential of the MCF7 cells were less than these parameters in the MDA-MB-231 cells and it is unknown why PAQR 3 expression and aggressiveness do not correlate, but it would be significant to investigate this in future studies. We also found that overexpression of the PAQR 3 gene inhibited the invasion and colony formation of breast cancer cells in vitro.

Furthermore, some recent studies have indicated that PAQR3 has a functional interaction with p53 in cancer formation and epithelial-to-mesenchymal transition (14). In addition, some data have provided convincing evidence that PAQR3 plays an important role in regulating obesity and energy homeostasis accompanied by modulation of leptin signaling (13). PAQR3 also modulates insulin sensitivity, energy metabolism, as well as obesity in mice partly via negative regulation of PI3K $(13,16)$. Moreover, it is likely that the reduced expression of PAQR3 in human tumors could relieve the inhibitory effect of PAQR 3 on histone H3 lysine 4 (H3K4) methylation, leading to facilitation of hypoxia-induced H3K4 methylation and activation of mesenchymal gene expression (17). 
There is increasing evidence showing that gene silencing due to aberrant DNA methylation is an early event in carcinogenesis and may serve as a potential diagnostic and prognostic biomarker in some cancers $(18,19)$. Recently, in humans, it was found that the expression level of PAQR3 was downregulated in many types of cancers including colorectal cancer, gastric cancer, osteosarcoma, laryngeal squamous cell carcinoma, liver cancer and breast cancer $(9,10,20-22)$. In the present study, MSP analysis showed that PAQR3 promoter methylation was significantly higher in breast cancer tissues than that in adjacent non-tumor tissues. In addition, bisulfite sequencing analysis of the $\mathrm{CpG}$ islands around the $\mathrm{PAQR} 3$ promoter was used to detect the methylation status, indicating dense methylation of $\mathrm{CpG}$ sites in breast cancer tissues when compared with adjacent non-tumor tissues. Further analysis showed that aberrant PAQR3 promoter methylation in breast cancer was associated with poor overall survival, which can provide important prognostic information for patients with breast cancer. These findings suggested that epigenetic silencing of the PAQR3 promoter via hypermethylation may be one of the major mechanisms for inactivation of this gene in breast cancer.

The recognition that silencing of tumor suppressor genes through promoter hypermethylation plays a significant role in tumorigenesis has led to the clinical use of hypomethylating agents including 5-Aza-dC (23), which has been approved for the treatment of cancer. In the present study, the results from qPCR and western blotting showed that 5-Aza-dC treatment significantly promoted expression of the PAQR3 gene at both the mRNA and protein levels in the MCF-7 cancer cell line in a dose-dependent manner with the highest expression at $10 \mu \mathrm{mol} / 1$, indicating that demethylation by 5 -Aza-dC contributed to the reactivation of PAQR3, thus resulting in a decrease in colony formation rate and the invasive capacity of the MCF-7 cells as detected using Transwell and colony formation assays. However, 5-Aza-dC demethylation is not specific. It can activate many other genes, thus 5 -Aza-dC may directly promote PAQR3 gene promoter demethylation, or it may act through the regulation of other genes that promote demethylation, indirectly mediating PAQR3 gene expression.

In conclusion, PAQR 3 promoter hypermethylation is observed in breast cancer, and promoter hypermethylation of PAQR3 is significantly correlated with poorer survival in breast cancer patients. Bearing these findings in mind, it will be important in the future to elucidate the mechanism underlying PAQR3 regulation on breast cancer cells. Further research is needed to determine the mechanism of promoter methylation of PAQR3 in breast cancer tumorigenesis.

\section{Acknowledgements}

This study was supported by the grants from the Jiangsu Postdoctoral Science Foundation (grant no. 1402202C).

\section{References}

1. Hinestrosa MC, Dickersin K, Klein P, Mayer M, Noss K, Slamon D, Sledge G and Visco FM: Shaping the future of biomarker research in breast cancer to ensure clinical relevance. Nat Rev Cancer 7: 309-315, 2007.
2. Liu Y, Li H, Feng J, Cui X, Huang W, Li Y, Su F, Liu Q, Zhu J, Lv X, et al: Lin28 induces epithelial-to-mesenchymal transition and stemness via downregulation of let-7a in breast cancer cells. PLoS One 8: e83083, 2013.

3. Dworkin AM, Huang TH and Toland AE: Epigenetic alterations in the breast: Implications for breast cancer detection, prognosis and treatment. Semin Cancer Biol 19: 165-171, 2009.

4. Feng L, Xie X, Ding Q, Luo X, He J, Fan F, Liu W, Wang Z and Chen Y: Spatial regulation of Raf kinase signaling by RKTG. Proc Natl Acad Sci USA 104: 14348-14353, 2007.

5. Luo X, Feng L, Jiang X, Xiao F, Wang Z, Feng GS and Chen Y: Characterization of the topology and functional domains of RKTG. Biochem J 414: 399-406, 2008.

6. Xie X, Zhang Y, Jiang Y, Liu W, Ma H, Wang Z and Chen Y: Suppressive function of RKTG on chemical carcinogen-induced skin carcinogenesis in mouse. Carcinogenesis 29: 1632-1638, 2008.

7. Zhang Y, Jiang X, Qin X, Ye D, Yi Z, Liu M, Bai O, W, Xie X, Wang Z, et al: RKTG inhibits angiogenesis by suppressing MAPK-mediated autocrine VEGF signaling and is downregulated in clear-cell renal cell carcinoma. Oncogene 29: 5404-5415, 2010

8. Fan F, Feng L, He J, Wang X, Jiang X, Zhang Y, Wang Z and Chen Y: RKTG sequesters B-Raf to the Golgi apparatus and inhibits the proliferation and tumorigenicity of human malignant melanoma cells. Carcinogenesis 29: 1157-1163, 2008.

9. Wang X, Li X, Fan F, Jiao S, Wang L, Zhu L, Pan Y, Wu G, Ling ZQ, Fang J, et al: PAQR3 plays a suppressive role in the tumorigenesis of colorectal cancers. Carcinogenesis 33: 2228-2235, 2012.

10. Ling ZQ, Guo W, Lu XX, Zhu X, Hong LL, Wang Z, Wang Z and Chen Y: A Golgi-specific protein PAQR3 is closely associated with the progression, metastasis and prognosis of human gastric cancers. Ann Oncol 25: 1363-1372, 2014.

11. Ma Z, Wang Y, Piao T, Li Z, Zhang H, Liu Z and Liu J: The tumor suppressor role of PAQR3 in osteosarcoma. Tumour Biol 36: 3319-3324, 2015.

12. Tang YT, Hu T, Arterburn M, Boyle B, Bright JM, Emtage PC and Funk WD: PAQR proteins: A novel membrane receptor family defined by an ancient 7-transmembrane pass motif. J Mol Evol 61: 372-380, 2005.

13. Wang L, Wang X, Li Z, Xia T, Zhu L, Liu B, Zhang Y, Xiao F, Pan Y, Liu Y, et al: PAQR3 has modulatory roles in obesity, energy metabolism, and leptin signaling. Endocrinology 154: 4525-4535, 2013

14. Jiang Y, Xie X, Li Z, Wang Z, Zhang Y, Ling ZQ, Pan Y, Wang Z and Chen Y: Functional cooperation of RKTG with p53 in tumorigenesis and epithelial-mesenchymal transition. Cancer Res 71: 2959-2968, 2011.

15. Yu X, Li Z, Chan MT and Wu WK: PAQR3: A novel tumor suppressor gene. Am J Cancer Res 5: 2562-2568, 2015.

16. Wang X, Wang L, Zhu L, Pan Y, Xiao F, Liu W, Wang Z, Guo F, Liu Y, Thomas WG, et al: PAQR3 modulates insulin signaling by shunting phosphoinositide 3-kinase p1 $10 \alpha$ to the Golgi apparatus. Diabetes 62: 444-456, 2013.

17. Liu C, Zhang Y, Hou Y, Shen L, Li Y, Guo W, Xu D, Liu G, Zhao Z, Man K, et al: PAQR3 modulates H3K4 trimethylation by spatial modulation of the regulatory subunits of COMPASS-like complexes in mammalian cells. Biochem J 467: 415-424, 2015.

18. Jones PA and Baylin SB: The epigenomics of cancer. Cell 128: 683-692, 2007.

19. Esteller M: Cancer epigenomics: DNA methylomes and histone-modification maps. Nat Rev Genet 8: 286-298, 2007.

20. Wu Q, Zhuang $\mathrm{K}$ and $\mathrm{Li} \mathrm{H}$ : PAQR3 plays a suppressive role in laryngeal squamous cell carcinoma. Tumour Biol 37: 561-565, 2016.

21. Wu HG, Zhang WJ, Ding Q, Peng G, Zou ZW, Liu T, Cao RB, Fei SJ, Li PC, Yang KY, et al: Identification of PAQR3 as a new candidate tumor suppressor in hepatocellular carcinoma. Oncol Rep 32: 2687-2695 2014.

22. Li Z, Ling Z-Q, Guo W, Lu XX, Pan Y, Wang Z and Chen Y: PAQR3 expression is downregulated in human breast cancers and correlated with HER2 expression. Oncotarget 6: 12357-12368, 2015.

23. Fenaux P: Inhibitors of DNA methylation: Beyond myelodysplastic syndromes. Nat Clin Pract Oncol 2 (Suppl 1): S36-S44, 2005. 